\title{
Novel standards and emerging therapies for systemically treatment-naïve clear cell renal cell carcinoma - a rapidly changing landscape
}

\author{
Ritesh R. Kotecha, Martin H. Voss \\ Department of Medicine, Memorial Sloan Kettering Cancer Center, New York, NY 10065, USA. \\ Correspondence to: Dr. Martin H. Voss, Department of Medicine, Memorial Sloan Kettering Cancer Center, 300 East 66th \\ Street, New York, NY 10065, USA. E-mail: vossm@mskcc.org \\ How to cite this article: Kotecha RR, Voss MH. Novel standards and emerging therapies for systemically treatment-naïve clear \\ cell renal cell carcinoma - a rapidly changing landscape. J Cancer Metastasis Treat 2021;7:67. https://dx.doi.org/10.20517/2394- \\ 4722.2021 .163
}

Received: 10 Aug 2021 First Decision: 14 Sep 2021 Revised: 19 Sep 2021 Accepted: 28 Oct 2021 Published: 5 Dec 2021

Academic Editor: Hendrik Van Poppel Copy Editor: Xi-Jun Chen Production Editor: Xi-Jun Chen

\begin{abstract}
The last decade has brought about major advances in systemic therapy for patients with advanced clear cell renal cell carcinoma. The introduction of angiogenesis targeting agents, immune checkpoint inhibitors, and combinations thereof has resulted in a multitude of therapeutic standards for patients with newly diagnosed advanced disease. With the rapid adoption and increasing number of available options for patients, selection amongst treatment regimens has become complex. Further, a new balance is also being sought to optimize treatment outcomes and limit treatment-related toxicities. With a rising bar against which novel therapeutics are being measured, the field looks toward an evolved understanding of tumor biology to help pave new ways forward for individualized therapy. Here, we review pivotal studies that led to regulatory approvals and ongoing clinical trials conducted in patients with systemically untreated clear cell renal cell carcinoma and provide perspective on how newly emerging data can be integrated into this rapidly changing landscape.
\end{abstract}

Keywords: Renal cell carcinoma, combination immunotherapy, clinical trials, first-line

\section{INTRODUCTION}

Over the last two decades, the clinical management of advanced clear cell renal cell carcinoma (ccRCC) has 
transformed significantly, and the serial introduction of novel classes of therapeutic agents has notably improved clinical outcomes over time. Earlier versions of immunotherapy in the form of recombinant cytokines constituted a standard in the 1990s, but their use was limited by poor tolerance, and only modest activity with sustained clinical benefit was achieved in a minority of patients ${ }^{[1]}$. Molecular targeting agents, including multi-tyrosine kinase inhibitors (TKIs) of the vascular endothelial growth factor (VEGF) receptor and mTOR pathway blockers, subsequently took their place as the standard of care for patients in the firstline setting and were typically given as single agents ${ }^{[2]}$. The introduction of contemporary immunotherapy in the form of immune checkpoint inhibitors (ICIs) initially occurred in pre-treated patients treated with PD-1 directed monotherapy ${ }^{[3]}$, but this quickly moved into the frontline setting where combination therapy pairing agents targeting PD-1 and CTLA-4 were shown to improve patient survival, freedom from progression and quality of life compared to the prior gold standard, single-agent TKI therapy ${ }^{[4]}$. Soon after, the introduction of combinations pairing the old with the new, TKI plus ICI therapy again demonstrated superior patient response and survival outcomes when compared to TKI alone. The advent of several new regimens approved over 2018-2021 has now ushered in a new treatment era, with combination therapy as the frontline standard for all eligible advanced ccRCC patients $s^{[5-8]}$. As all these novel regimens have demonstrated superiority over TKI alone, but none have been compared to one another, selection of a preferred contemporary standard for clinical practice or as the comparator on new prospective trials remains complex. Lastly, while SURTIME ${ }^{[0]}$ and CARMENA ${ }^{[10]}$ investigated the role of cytoreductive nephrectomy with sunitinib, these studies provide complementary data and inform that many patients will start with immediate upfront systemic therapy with subsequent consideration of cytoreduction if clinical circumstances permit. Here, we review currently approved treatment regimens and overall treatment strategies for patients systemically treatment-naïve ccRCC.

\section{COMBINATION THERAPY IS THE FRONTLINE STANDARD FOR ADVANCED CCRCC}

For many years the routine assessment of patients with metastatic ccRCC initiating first-line systemic therapy integrated clinical prognostic tools like the Memorial Sloan Kettering Cancer Center (MSKCC) and the International Metastatic RCC Database Consortium (IMDC) risk classification system ${ }^{[1,12]}$. Developed during the cytokine and targeted therapy eras, respectively, these programs integrate clinical and laboratory data to group patients into favorable, intermediate, and poor-risk strata. However, for years, these tools were merely appreciated (and serially validated) for their usefulness in informing disease prognosis, and registration strategies in the metastatic setting rarely focused on specific risk categories ${ }^{[13,14]}$. More recent data highlight that these risk classifiers serve as clinical surrogates for underlying tumor biology, with RCC tumors characterized across different disease phenotypes, including enrichment of upregulation of tumor angiogenesis in favorable risk patients $v s$. proliferative/inflammatory profiles in patients with intermediate/poor-risk disease ${ }^{[15-17]}$. This has enabled a more nuanced understanding of how risk status (and underlying biology) may be informative for choice of therapy in previously systemically untreated ccRCC patients.

\section{COMBINATION IMMUNE CHECKPOINT BLOCKADE THERAPY}

The first modern combination therapy proven to be superior to TKI monotherapy was ipilimumab plus nivolumab on the CheckMate-214 trial $^{[4]}$. Extended follow-up analyses of this global phase 3 study with a dedicated focus on IMDC intermediate/poor-risk patients confirm superior clinical outcomes over singleagent sunitinib for the three co-primary endpoints: overall response rate (ORR), progression-free survival (PFS, HR $=0.73$ ), and overall survival $(\mathrm{OS}, \mathrm{HR}=0.68)(\text { see Table } 1)^{[18,19]}$. In the updated 4-year and recently presented 5-year survival analyses, there is also a better appreciation for the long-term clinical benefit from therapy in those patients who achieve radiographic response to therapy. For instance, of those patients who achieved a complete response (CR), $32 \%$ of patients continue to remain on systemic therapy, and $46 \%$ of 
Table 1. Summary of approved combination therapytrials for systemically untreated ccRCC patients

\begin{tabular}{|c|c|c|c|c|c|c|}
\hline & \multicolumn{2}{|c|}{ CheckMate-214^ } & KEYNOTE-426* & Javelin Renal-101 & CheckMate 9ER & CLEAR \\
\hline & \multicolumn{2}{|c|}{ Ipilimumab + Nivolumab } & Axitinib + Pembrolizumab & Axitinib + Avelumab & Cabozantinib + Nivolumab & Lenvatinib + Pembrolizumab \\
\hline Median follow-up & \multicolumn{2}{|l|}{67.7 months } & 42.8 months & 11.6 months & 18.1 months & 26.6 months \\
\hline Total patients & \multicolumn{2}{|l|}{1096} & 861 & 560 & 651 & 1069 \\
\hline $\begin{array}{l}\text { IMDC } \\
\text { Fav/Int/Poor }\end{array}$ & \multicolumn{2}{|l|}{$23 / 61 / 17$} & $31.9 / 55.1 / 13$ & $19.3 / 64.1 / 16.3$ & $22.6 / 57.6 / 19.7$ & $31 / 59.2 / 9.3$ \\
\hline Sarcomatoid features (\%) & \multicolumn{2}{|l|}{13.2} & 17.9 & 12.2 & 11.5 & 7.9 \\
\hline Nephrectomy status (\%) & \multicolumn{2}{|l|}{82} & 82.6 & 83 & 68.7 & 73.8 \\
\hline \multirow[t]{2}{*}{ ORR (\%) } & Int/Poor & ITT & \multirow{2}{*}{60.4} & PD-L1+ & \multirow{2}{*}{55.7} & \multirow{2}{*}{71} \\
\hline & 42 & 39 & & 55.2 & & \\
\hline CR (\%) & 11 & 12 & 10 & 5.6 & 8 & 16.1 \\
\hline PD (\%) & 19.3 & 17.6 & 11.3 & 11.5 & 5.6 & 5.4 \\
\hline Median PFS (months) & 12 & 12 & 15.7 & 13.8 & 16.6 & 23.9 \\
\hline PFS HR (Cl) & $0.73(0.61-0.87)$ & $0.86(0.73-1.01)$ & $0.68(0.58-0.80)$ & $0.61(0.47-0.79)$ & $0.51(0.41-0.64)$ & $0.39(0.32-0.49)$ \\
\hline OS HR (Cl) & $0.68(0.58-0.81)$ & $0.72(0.62-0.85)$ & $0.73(0.60-0.88)$ & $0.82(0.53-1.28)$ & $0.6(0.4-0.89)$ & $0.66(0.49-0.88)$ \\
\hline
\end{tabular}

*Extended follow-up. IMDC: International Metastatic RCC Database Consortium; ORR: objective response rate; CR: complete response; PD: progressive disease; PFS: progression-free survival; HR: hazard ratio; CI: confidence interval; OS: overall survival; ITT: intention to treat.

patients have discontinued treatment without needing a subsequent therapy ${ }^{[18,19]}$. Early treatment failure was not uncommon with a landmark PFS at 18 months being $42 \%$ and progressive disease (PD) being the best response in $20 \%$ of patients. However, progression events became notably less likely beyond the 2 -year mark with 30-month and 42-month landmark PFS rates nearly identical at 35\% and 33\%, respectively. For those patients who sustained response at 3 years, the probability of remaining in response for an additional 2 years was $89 \%$ (ITT population) and 90\% (intermediate/poor risk population) ${ }^{[18,19]}$. This "tailof-the-curve" phenomenon, together with the observed response durability and the marvel of treatment-free survival intervals, highlight the draw to immunotherapy, particularly in contrast to the waning responses and enduring toxicities usually seen with chronic TKI therapy. In patients with IMDC favorable risk disease (not part of the primary efficacy analysis pre-specified in the trial's statistical analysis plan), ORR, PFS, and even landmark OS analyses numerically favored the sunitinib arm on this trial; hence, the FDA label is limited to patients with intermediate/poor disease. Interestingly, complete responses were observed across all IMDC risk groups - $11 \%$ for intermediate-poor risk patients and 13\% for favorable risk patients, and responses were more durable on the investigational arm, regardless of IMDC risk status ${ }^{[18]}$. The unique safety profile of an all-ICI regimen is not as well characterized by adverse event assessment (by CTCAE); instead, the fact that $29 \%$ of patients on the ipilimumab/nivolumab arm required high-dose corticosteroids for the treatment of adverse events, including $10 \%$ for $30+$ days speaks to the unique challenges of managing the immune-mediated toxicities of this regimen. Notably, there remains no data to support that steroid use impacts the efficacy of this regimen. With attention to histologic variants, post-hoc analyses further show that in 
RCC tumors with sarcomatoid features, high response rates and survival outcomes can also be achieved with combination ipilimumab and nivolumab ${ }^{[20]}$. In this population, the confirmed ORR was $60.8 \%$ with notably $18.9 \%$ of patients achieving a complete response, highlighting a significant step forward for this patient subgroup with a historically poor prognosis.

\section{COMBINATION ANTI-ANGIOGENESIS AND IMMUNE CHECKPOINT BLOCKADE THERAPY}

The increasing number of different VEGF and ICI therapy permutations has provided valuable lessons on careful drug selection and dosing of paired agents. Conceptually, many VEGF targeting therapies with established roles in the monotherapy setting have favorable downstream immunomodulatory effects on the RCC suppressive tumor microenvironment. Furthermore, with toxicity profiles that are mechanistically non-overlapping with ICI therapy, these should, in principle, all lend themselves well to combination strategy ${ }^{[2]}$. However, a steadily increasing number of trials studying various TKI/ICI combinations have revealed stark differences in efficacy and toxicity patterns, which emphasizes the importance of agent selection. For instance, the pairing of sunitinib or pazopanib with ICI therapy was met with unacceptably high rates of toxicity, with $39.4 \%$ and $80 \%$ of patients discontinued nivolumab plus sunitinib and pembrolizumab plus pazopanib, respectively ${ }^{[22,23]}$. In addition, certain regimens, including axitinib with avelumab (JAVELIN-Renal-101) and bevacizumab with atezolizumab (IMmotion-151), improved upon radiographic responses achieved with TKI therapy alone but did not appear to carry an OS advantage ${ }^{[8,24]}$. Amongst these reported combinations, the three that have demonstrated level 1 evidence of unequivocal superiority over TKI monotherapy include axitinib with pembrolizumab, cabozantinib with nivolumab, and lenvatinib with pembrolizumab.

Reported extended follow-up of KeyNote-426, the global phase III study of axitinib and pembrolizumab vs. sunitinib, continues to confirm the significant clinical benefit for patients with systemically untreated ccRCC with respect to dual primary endpoints PFS and OS, with HR of 0.73 and 0.68 , respectively (see Table 1$)^{[25,26]}$. ORR as a key secondary endpoint also favored the combination, and patients treated with combination axitinib and pembrolizumab achieved an ORR of $60 \%$, of which $10 \%$ sustained a CR. The superiority of the investigational arm was apparent early on this trial, and in the extended follow-up, $83 \%$ of patients sustained some measure of tumor reduction, and the rate of PD as the best response was only $11 \%$ with combination therapy. The primary analysis plan was neither specific to any IMDC risk category nor tumor PD-L1 status, and superiority was apparent across all such groups on secondary analyses. In a recent update, $29.9 \%$ of enrolled participants have even completed at least 2 years of systemic therapy, and of those, $14 \%$ achieved a $\mathrm{CR}^{[27]}$. In the CheckMate $9 \mathrm{ER}$ study, cabozantinib combined with nivolumab was compared to sunitinib monotherapy and demonstrated superior PFS, the primary endpoint of the trial (see Table 1). Secondary endpoints ORR and OS were also notably improved for cabozantinib/nivolumab ${ }^{[6]}$. At a median follow-up of approximately 18 months, combination therapy had an ORR of $55.7 \%$, with $8 \%$ of patients achieving a complete response. Further, superior OS ( $\mathrm{HR}=0.6)$ was found regardless of PD-L1 expression and across all IMDC risk groups, and emerging data also confirms that this clinical benefit is extended for patients with sarcomatoid $\mathrm{RCC}^{[28]}$. Based on the data from these pivotal studies, both axitinib/pembrolizumab and cabozantinib/nivolumab have received FDA approval and remain standard options in the first-line setting for all eligible advanced ccRCC patients. In contrast to ipilimumab/nivolumab, their registration is not tied to a specific IMDC/MSKCC risk status.

The most recently reported TKI/ICI combination to gain FDA approval was gathered on the CLEAR study, a three-arm trial which randomized patients 1:1:1 to the TKI/ICI combination of lenvatinib plus pembrolizumab $v s$. the non-ICI-based combination of lenvatinib plus everolimus $v s$. TKI monotherapy with standard sunitinib ${ }^{[7]}$. In this phase III global clinical trial, which tested PFS with each combination $v s$. 
sunitinib as the primary endpoint, the study indeed confirmed that patients treated with either lenvatinib combination (pembrolizumab or everolimus) achieved significantly prolonged PFS compared to sunitinib alone $(\mathrm{HR}=0.39$ for pembrolizumab combination and $\mathrm{HR}=0.65$ for everolimus combination, respectively, see also Table 1). In addition, patients treated with lenvatinib/pembrolizumab demonstrated superior OS compared to sunitinib $(\mathrm{HR}=0.66)$, with a high overall response rate $(71 \%)$, notable CR rate $(16.1 \%)$, and low rate of primary PD (5.4\%). The OS for patients treated with lenvatinib/everolimus was not found to be superior to sunitinib $(\mathrm{HR}=1.15)$. Subgroup analyses support that the OS benefit seen for combination lenvatinib and pembrolizumab over sunitinib likely extends to those patients with PD-L1 negative tumors, but this could not be confirmed in patients with favorable risk disease.

In all ICI/TKI trials, safety and tolerance observed for these above regimens listed here were largely in keeping with the adverse event profiles typically seen with each of the individual agents without the sense that an amplified toxicity signal emerged as being dose-limiting. Individual regimens varied in the reported incidence of hepatic and GI toxicity, which may be related to mechanisms of action, drug interaction, differences in the starting dose, or all the above.

All combinations described above have expanded the number of combination options available to patients with advanced disease and increased the complexity of individualizing care. The choice between an ICI doublet (ipilimumab/nivolumab) and one of the various TKI/ICI combinations may be driven by: (1) IMDC risk status (as ipilimumab/nivolumab being solely approved in intermediate/poor risk patients); (2) a notably lower upfront failure rate for all TKI/ICI combinations (explained by dual mechanism of action and relevant in patients with rapidly progressive, symptomatic disease who may not reach second-line therapy for which the risk remains higher with ipilimumab/nivolumab $)^{[2]]}$; (3) the above mentioned PFS "tail of the curve" for ipilimumab/nivolumab (which should motivate consideration of this regimen in patients with no/little concern for rapid progression and deterioration, until TKI/IO datasets are more mature and can speak to the presence of absence of a tail of the curve for those regimens); and (4) co-morbidities (uncontrolled cardiovascular disease arguing against TKI-containing regimen; conditions limiting tolerability of high-dose corticosteroids causing concern for use of the ICI doublet, which comes with a notably higher risk of requiring steroids for high-risk immune related toxicity). Once the determination is made to proceed with TKI/ICI, treating physicians are left with several regimens that have met all primary endpoints during the registration process. Study populations for KeyNote-426, CheckMate 9ER and CLEAR varied notably, and a clear "winner" cannot be chosen while follow-up is comparatively short for all of these, and the "tail-of-the-curve" question is left unanswered still. Instead, the decision will be driven by physician experience with and their preference for individual agents, reported starting doses (e.g., cabozantinib is started at an attenuated dose with nivolumab; axitinib is started at a standard dose with pembrolizumab; lenvatinib is started at a higher dose with pembrolizumab compared to the previously approved combination with everolimus) and the individual nuances of toxicity that may be particularly enticing with each (e.g., low rate of hepatic events with lenvatinib and pembrolizumab or low rate of dose reductions needed for cabozantinib with nivolumab).

\section{OPTIMIZING ICI THERAPY - CAN WE ACHIEVE MORE WITH LESS?}

Deep responses and durable anticancer effects have been observed in those patients receiving ICI monotherapy - albeit in a lesser proportion of patients ${ }^{[30]}$. Results from KeyNote-427 Cohort A, a phase II study of pembrolizumab monotherapy in systemically untreated ccRCC patients, demonstrated an ORR of $36.4 \%$, of which $3 \%$ of patients also achieved a complete response and $15 \%$ of patients achieved a high depth of response with $>80 \%$ tumor reduction ${ }^{[30]}$. Such ICI monotherapy comes at a notably lower risk for highgrade toxicity than ICI-containing combination therapy. Several groups have investigated adaptive designed 
trials which escalate and de-escalate immunotherapy based upon an individualized response to minimize risk while attempting to achieve durable benefit.

Three previously reported adaptive trials (TITAN, OMNIVORE, HCRN-GU-260) had explored a novel stepwise scheme of adjusting the intensity of ICI therapy per radiographic response. All three studies enrolled ICI-naïve patients with clear cell histology and initially started with the anti-PD1 monotherapy nivolumab. Subsequently, per response assessment during the initial months of therapy, treatment was matched to the degree of therapeutic benefit observed, incorporating varying strategies with de-escalation (to active surveillance) $v s$. intensification (with the addition of ipilimumab) $v s$. continuation of nivolumab alone. The three studies varied in study population (distribution of risk status and prior treatment exposure), length of nivolumab induction and the type of risk adaptation escalation; hence, the results must be viewed within the context of the individual study design. For instance, nearly half the patients on TITAN had received prior therapy, and radiographic responses in the first 4 months were achieved in $22.7 \%$ of patients. At that point, those with SD or PD received up to 4 cycles of ipilimumab/nivolumab with additional responses observed in $12 \%$ of previous non-responders $(n=12 / 104 \text { patients })^{[31]}$. In OMNIVORE, where half the patients had received TKI therapy, patients were initially treated with nivolumab monotherapy for up to 6 months; those who achieved a response had treatment discontinued altogether, while those who did not were treated with 2 cycles of ipilimumab ${ }^{[32]}$. The 6 -month ORR was $14 \%$, and 5 patients sustained a treatment-free interval for more than a year. For non-responders, the addition of 2 cycles of ipilimumab for salvage therapy applied after 6 months led to a response rate of only $4 \%(n=2 / 57$ patients). Lastly, in the HCRN-GU-260 study, all patients were treatment-naïve and underwent therapy with escalating doses of nivolumab (240 mg $\times 6$ doses, $360 \mathrm{mg} \times 4$ doses, and $480 \mathrm{mg}$ monthly). Patients who experienced disease progression prior to or stable disease at 48 weeks were then offered salvage ipilimumab and nivolumab for 4 cycles $^{[33]}$. In this schema, induction nivolumab was associated with PRs and CRs of $26 \%$ and $5.7 \%$ of patients, respectively (ORR $=31.7 \%$ ), and the addition of salvage ipilimumab led to an added response in 4/30 patients (13.3\%). Expectedly, high-grade immune-mediated adverse events were more frequent during the salvage ipilimumab portion of each study, but data in terms of escalating toxicities with the additional ipilimumab for those who sustained minor adverse events has not been reported. The relatively small number of patients whose treatment was escalated/de-escalated on these trials make it challenging to appreciate the nuances of how patients fair with individualized treatment intensity. Nonetheless, one can make valuable observations across all three studies. First, the addition of ipilimumab to nivolumab monotherapy could convert non-response to at least PR in all three trials, albeit in only a small proportion of patients (4\%-13\% of patients); and second, the composite ORR across all treated patients receiving individualized treatment on these three trials, and particularly the reported CR rates raise concern that the sequence of PD-1 to combination CTLA- 4 directed combination therapy comes at the price of lost efficacy. As we await ongoing prospective efforts to formally assess the differences between ipilimumab plus nivolumab vs. nivolumab alone in the systemically untreated setting (NCT03873402), the low conversion and CR rates on OMNIVORE, TITAN and HCRN-GU-260 argue against the broad use of sequenced nivolumab/ipilimumab and support the use of combination therapy in the upfront setting.

Another effort is accepting upfront ipilimumab/nivolumab as the standard frontline regimen but tailoring treatment beyond the initial imaging assessments. For example, in the pivotal phase III PDIGREE study (NCT03793166), systemically untreated IMDC intermediate-poor risk ccRCC patients undergo treatment with induction ipilimumab and nivolumab therapy per standard schedule. Those who sustain PD are shifted towards cabozantinib monotherapy. Patients who achieve a CR to induction ipilimumab/nivolumab continue standard nivolumab maintenance up to 1 year. Patients who achieve a partial response or stable disease are randomized to receive nivolumab monotherapy or combination cabozantinib/nivolumab 
therapy. As these data remain forthcoming, understanding the optimal sequence of these combinations and other ongoing efforts will be critical to put this data within the context of cabozantinib/nivolumab in the first-line setting and increasing data supportive of combination therapy in the post-ICI space ${ }^{[34]}$. Lastly, in a similar vein in favor of de-escalation, a discontinuation design study where patients treated with axitinib and avelumab discontinue axitinib if sustaining a response within the first 36 weeks of therapy also may provide perspective on these types of adaptive strategies (TIDE-A, NCT04698213).

Towards an individualized designed study using a pre-therapy biomarker for therapy selection, the BIONIKK study (NCT02960906) was recently presented ${ }^{[35]}$. In this innovative clinical trial, tumor tissue submitted at study entry was analyzed to distinguish participating systemically untreated ccRCC patients into four molecularly defined groups $(\mathrm{ccRCC} 1-4)$ based on previously defined transcriptomic features ${ }^{[36]}$. Molecular classification then determined treatment allocation - patients with ccRCC1 (immune-low) and ccRCC4 (immune-high) were randomized between nivolumab or ipilimumab and nivolumab, and patients with ccRCC2 (angiogenesis-high) and ccRCC3 (normal-like) tumor signatures were randomized between ipilimumab and nivolumab or TKI therapy with sunitinib or pazopanib. In these groups, no significant differences were observed in terms of the primary endpoint of treatment response, but combination ipilimumab and nivolumab was numerically higher in the ccRCC1 group (immune-low) ${ }^{[35]}$. While the results remain preliminary, this study notably provides a proof of concept that molecular stratification to target individual tumor biology rationally is feasible and provides initial foundational steps toward precision medicine approaches integrating angiogenesis targeting, immune therapy, and other novel treatment approaches.

\section{ESCALATING BEYOND TKI/ICI THERAPY - CAN WE ACHIEVE MORE WITH MORE?}

With recent advances having newly yet firmly established doublet combinations as the current standard of care, multiple ongoing investigations are taking combination therapy one step further and testing triplet regimens for the first-line treatment of ccRCC [Table 2]. One of the most notable ongoing studies is the pivotal global phase III COSMIC-313 study (NCT03937219), which randomizes patients with IMDC intermediate-poor risk disease to receive standard ipilimumab/nivolumab with either cabozantinib or placebo ${ }^{[37]}$. After the initial induction phase, patients receive nivolumab (for a maximum of 2 years) and cabozantinib or placebo. While encouraging anti-tumor efficacy and tolerability has been reported in phase I studies ${ }^{[38]}$, it will be important to note specific and chronic toxicities, particularly in the context of the reported cabozantinib/nivolumab therapy data.

Novel agents that have also shown efficacy in the first-line setting are also being positioned in the triplicate space, with the incorporation of a VEGFR TKI and ICI backbone. For example, belzutifan (MK-6482) is a potent and selective orally available HIF-2 $\alpha$ inhibitor that has received breakthrough designation for the treatment of VHL associated RCC in patients with non-metastatic tumors. In the phase II setting, encouraging therapeutic responses have been reported with monotherapy (NCT04195750) and in combination with cabozantinib (NCT03634540) $)^{[39]}$. In line with this, evaluation in the triplicate setting is now being pursued on a global phase III study (NCT04736706). Here, systemically untreated patients are randomized to receive lenvatinib/pembrolizumab, lenvatinib/pembrolizumab/quavonlimab (an anti-CTLA4 antibody), or lenvatinib/pembrolizumab/belzutifan. This study will relevantly shed light on the combination of VEGF, ICI, and HIF-2 $\alpha$ inhibition and will also notably provide data on a competing triplicate combination given the arm, including lenvatinib with dual ICI approach (similar to COSMIC313). 
Table 2. Select phase III ongoing clinical trials for systemically treatment-naïve RCC

\begin{tabular}{|c|c|c|}
\hline Treatment regimen & Comparator & $\begin{array}{l}\text { Clinical trial } \\
\text { information }\end{array}$ \\
\hline Ipilimumab + Nivolumab & Nivolumab & $\begin{array}{l}\text { CA209-8Y8, } \\
\text { NCT03873402 }\end{array}$ \\
\hline $\begin{array}{l}\text { Ipilimumab + Nivolumab, followed by Nivolumab (CR), } \\
\text { Cabozantinib + Nivolumab/Nivolumab (SD/PR), or Cabozantinib } \\
\text { (PD) }\end{array}$ & $\begin{array}{l}\text { *Select patients only (non-CR, non-PD to } \\
\text { induction with Ipilimumab + Nivolumab): } \\
\text { Nivolumab vs. Nivolumab + Cabozantinib }\end{array}$ & PDIGREE, NCT03793166 \\
\hline Cabozaninib + Ipilimumab + Nivolumab & Ipilimumab + Nivolumab & $\begin{array}{l}\text { COSMIC-313, } \\
\text { NCT03937219 }\end{array}$ \\
\hline $\begin{array}{l}\text { Lenvatinib + Pembrolizumab + Belzutifan } \\
\text { Lenvatinib + Pembrolizumab + Quavonlimab }\end{array}$ & Lenvatinib + Pembrolizumab & $\begin{array}{l}\text { MK6482-012, } \\
\text { NCT04736706 }\end{array}$ \\
\hline Bempegaldesluekin + Nivolumab & Sunitinib or Cabozantinib & $\begin{array}{l}\text { PIVOT-09, } \\
\text { NCT03729245 }\end{array}$ \\
\hline $\begin{array}{l}\text { Systemic therapy (Ipilimumab + Nivolumab, Axitinib + } \\
\text { Pembrolizumab, or Axitinib + Avelumab) followed by cytoreductive } \\
\text { nephrectomy based upon response (PR/SD) }\end{array}$ & Continued systemic therapy & $\begin{array}{l}\text { PROBE/SWOG1931, } \\
\text { NCT04510597 }\end{array}$ \\
\hline
\end{tabular}

*Adaptive trial design: all patients are treated with ipilimumab + nivolumab initially, then assigned to treatment arms per their treatment response: PD - cabozantinib monotherapy; CR - nivolumab maintenance, to complete 1 year; non-CR/non-PD: randomization nivolumab vs. nivolumab + cabozantinib. CR: Complete response; PR: partial response; SD: stable disease; PD: progressive disease.

Building upon this framework, other promising therapeutic agents with strong clinical activity in the treatment-refractory setting are also being poised for a position in the triplicate space with combinations still under early investigation. For example, bempegaldesleukin (NKTR-214) is a pegylated first-in-class IL-2 receptor that potently binds to the CD122 surface to stimulate proliferation and mobilization into the tumor microenvironment ${ }^{[40]}$. Bempegaldesleukin was previously tested in a phase I/II study in patients with systemically treatment-naïve ccRCC and was found to have an ORR of 64\% (7/11 patients) with responses seen regardless of PD-L1 expression ${ }^{[4]}$. Based upon these encouraging results, a global phase III study of bempegaldesleukin in combination with nivolumab $v s$. investigator's choice TKI (sunitinib or cabozantinib) remains ongoing with results forthcoming (NCT03729245) ${ }^{[42]}$. In addition to this, an early phase I/II study (NCT04540705) tests this novel agent in combination with ICI and VEGFR TKI therapy (axitinib and cabozantinib, on separate arms) in the treatment-naïve setting. Other agents, including MEDI5752, a monovalent bispecific antibody co-targeting PD-1 and CTLA-4, paired with axitinib, similarly explore the triplicate combination of the mechanism of action for therapy of TKI and PD-1/CTLA-4 inhibition $(\mathrm{NCT} 04522323)^{[43]}$. As more triplicate combinations are developed, it will be helpful to discriminate these additional strategies within the known context of angiogenesis and immune activation to optimize synergism and limit overlapping toxicities.

\section{CONCLUSION}

The treatment paradigm for patients with systemically untreated ccRCC has significantly evolved, and multiple ICI-based combination therapies are the new standard for all eligible patients. Current first-line combinations have entered the arena in rapid succession, and while it is increasingly difficult to select amongst these available options, certain clinical situations and the clinician's treating experience may guide the selection of one regimen over another. Doublet therapy has become the standard comparator for clinical trials, but regimen choice will likely remain investigator-dependent and within the context of prognostic risk stratification. As triplicate regimens become a new reality, management of treatment-related adverse events will also become increasingly complex, and a key question is whether an achieved gain in efficacy justifies added toxicity. All this constitutes an urgent call for biomarker-driven studies and innovative trial designs with an overarching goal to incorporate molecular profiling and identify determinants for response, resistance, and ideally adverse effects, with adaptive study designs that offer the ability to intensify and de- 
escalate treatment for optimized antitumor effect and tolerance. As contemporary studies feed into this framework through exploration of additional biological axes pertinent for RCC biology and therapeutic effects, a new era for enhancing these combinations even further may soon come within reach.

\section{DECLARATIONS}

\section{Authors' contributions}

Writing, reviewing, and editing: Kotecha RR, Voss MH

\section{Availability of data and materials}

Not applicable.

\section{Financial support and sponsorship}

None.

\section{Conflicts of interest}

Kotecha RR has served as a consultant/advisory board member for Eisai, and reports receiving institutional research support from Pfizer and Takeda. Voss MH reports receiving commercial research support from Bristol-Myers Squibb, Pfizer and Genentech/Roche; honoraria from Novartis and Bristol-Myers Squibb; travel/accommodation from Astra Zeneca, Eisai, Novartis and Takeda; consultant/advisory board member for Aveo, Calithera Biosciences, Corvus Pharmaceuticals, Exelixis, Eisai, Merck, Onquality Pharmaceuticals, Novartis and Pfizer.

\section{Ethical approval and consent to participate \\ Not applicable.}

\section{Consent for publication}

Not applicable.

\section{Copyright}

(c) The Author(s) 2021.

\section{REFERENCES}

1. Negrier S, Escudier B, Lasset C, et al. Recombinant human interleukin-2, recombinant human interferon alfa-2a, or both in metastatic renal-cell carcinoma. Groupe Français d'Immunothérapie. N Engl J Med 1998;338:1272-8. DOI PubMed

2. Motzer RJ, Hutson TE, Cella D, et al. Pazopanib versus sunitinib in metastatic renal-cell carcinoma. N Engl J Med 2013;369:722-31. DOI PubMed

3. Motzer RJ, Escudier B, McDermott DF, et al; CheckMate 025 Investigators. Nivolumab versus everolimus in advanced renal-cell carcinoma. N Engl J Med 2015;373:1803-13. DOI PubMed PMC

4. Motzer RJ, Tannir NM, McDermott DF, et al; CheckMate 214 Investigators. Nivolumab plus ipilimumab versus sunitinib in advanced renal-cell carcinoma. N Engl J Med 2018;378:1277-90. DOI PubMed PMC

5. Rini BI, Plimack ER, Stus V, et al; KEYNOTE-426 Investigators. Pembrolizumab plus axitinib versus sunitinib for advanced renalcell carcinoma. N Engl J Med 2019;380:1116-27. DOI PubMed

6. Choueiri TK, Powles T, Burotto M, et al; CheckMate 9ER Investigators. Nivolumab plus cabozantinib versus sunitinib for advanced renal-cell carcinoma. N Engl J Med 2021;384:829-41. DOI PubMed PMC

7. Motzer R, Alekseev B, Rha SY, et al; CLEAR Trial Investigators. Lenvatinib plus pembrolizumab or everolimus for advanced renal cell carcinoma. N Engl J Med 2021;384:1289-300. DOI PubMed

8. Motzer RJ, Penkov K, Haanen J, et al. Avelumab plus axitinib versus sunitinib for advanced renal-cell carcinoma. $N$ Engl $J$ Med 2019;380:1103-15. DOI PubMed PMC

9. Bex A, Mulders P, Jewett M, et al. Comparison of immediate vs deferred cytoreductive nephrectomy in patients with synchronous metastatic renal cell carcinoma receiving sunitinib: the SURTIME randomized clinical trial. JAMA Oncol 2019;5:164-70. DOI PubMed PMC

10. Méjean A, Ravaud A, Thezenas S, et al. Sunitinib alone or after nephrectomy in metastatic renal-cell carcinoma. $N$ Engl $J$ Med 2018;379:417-27. DOI PubMed

11. Heng DY, Xie W, Regan MM, et al. Prognostic factors for overall survival in patients with metastatic renal cell carcinoma treated with 
vascular endothelial growth factor-targeted agents: results from a large, multicenter study. J Clin Oncol 2009;27:5794-9. DOI PubMed

12. Motzer RJ, Bacik J, Murphy BA, Russo P, Mazumdar M. Interferon-alfa as a comparative treatment for clinical trials of new therapies against advanced renal cell carcinoma. J Clin Oncol 2002;20:289-96. DOI PubMed

13. Choueiri TK, Halabi S, Sanford BL, et al. Cabozantinib versus sunitinib as initial targeted therapy for patients with metastatic renal cell carcinoma of poor or intermediate risk: the alliance A031203 CABOSUN trial. J Clin Oncol 2017;35:591-7. DOI PubMed PMC

14. Hudes G, Carducci M, Tomczak P, et al; Global ARCC Trial. Temsirolimus, interferon alfa, or both for advanced renal-cell carcinoma. N Engl J Med 2007;356:2271-81. DOI PubMed

15. Motzer RJ, Banchereau R, Hamidi H, et al. Molecular subsets in renal cancer determine outcome to checkpoint and angiogenesis blockade. Cancer Cell 2020;38:803-17.e4. DOI PubMed PMC

16. McDermott DF, Huseni MA, Atkins MB, et al. Clinical activity and molecular correlates of response to atezolizumab alone or in combination with bevacizumab versus sunitinib in renal cell carcinoma. Nat Med 2018;24:749-57. DOI PubMed PMC

17. Hakimi AA, Voss MH, Kuo F, et al. Transcriptomic profiling of the tumor microenvironment reveals distinct subgroups of clear cell renal cell cancer: data from a randomized phase III trial. Cancer Discov 2019;9:510-25. DOI PubMed PMC

18. Motzer R, Tannir N, Mcdermott D, et al. 661P Conditional survival and 5-year follow-up in CheckMate 214: First-line nivolumab + ipilimumab (N+I) versus sunitinib (S) in advanced renal cell carcinoma (aRCC). Ann Oncol 2021;32:S685-7. DOI

19. Albiges L, Tannir NM, Burotto M, et al. Nivolumab plus ipilimumab versus sunitinib for first-line treatment of advanced renal cell carcinoma: extended 4-year follow-up of the phase III CheckMate 214 trial. ESMO Open 2020;5:e001079. DOI PubMed PMC

20. Tannir NM, Signoretti S, Choueiri TK, et al. Efficacy and safety of nivolumab plus ipilimumab versus sunitinib in first-line treatment of patients with advanced sarcomatoid renal cell carcinoma. Clin Cancer Res 2021;27:78-86. DOI PubMed

21. Escudier B, Pluzanska A, Koralewski P, et al. Bevacizumab plus interferon alfa-2a for treatment of metastatic renal cell carcinoma: a randomised, double-blind phase III trial. Lancet 2007;370:2103-11. DOI PubMed

22. Amin A, Plimack ER, Ernstoff MS, et al. Safety and efficacy of nivolumab in combination with sunitinib or pazopanib in advanced or metastatic renal cell carcinoma: the CheckMate 016 study. J Immunother Cancer 2018;6:109. DOI PubMed PMC

23. Chowdhury S, Mcdermott DF, Voss MH, et al. A phase I/II study to assess the safety and efficacy of pazopanib (PAZ) and pembrolizumab (PEM) in patients (pts) with advanced renal cell carcinoma (aRCC). J Clin Oncol 2017;35:4506. DOI

24. Rini BI, Powles T, Atkins MB, et al. Atezolizumab plus bevacizumab versus sunitinib in patients with previously untreated metastatic renal cell carcinoma (IMmotion151): a multicentre, open-label, phase 3, randomised controlled trial. Lancet 2019;393:2404-15. DOI PubMed

25. Powles T, Plimack ER, Soulières D, et al. Pembrolizumab plus axitinib versus sunitinib monotherapy as first-line treatment of advanced renal cell carcinoma (KEYNOTE-426): extended follow-up from a randomised, open-label, phase 3 trial. Lancet Oncol 2020;21:1563-73. DOI PubMed

26. Rini BI, Plimack ER, Stus V, et al. Pembrolizumab (pembro) plus axitinib (axi) versus sunitinib as first-line therapy for advanced clear cell renal cell carcinoma (ccRCC): results from 42-month follow-up of KEYNOTE-426. J Clin Oncol 2021;39:4500. DOI

27. Plimack ER, Powles T, Bedke J, et al. Outcomes for patients in the pembrolizumab+axitinib arm with advanced renal cell carcinoma (RCC) who completed two years of treatment in the phase III KEYNOTE-426 study. J Clin Oncol 2021;39:327. DOI

28. Motzer RJ, Choueiri TK, Powles T, et al. Nivolumab + cabozantinib (NIVO+CABO) versus sunitinib (SUN) for advanced renal cell carcinoma (aRCC): Outcomes by sarcomatoid histology and updated trial results with extended follow-up of CheckMate 9ER. J Clin Oncol 2021;39:308. DOI

29. Alhalabi O, Hasanov E, Wilson NR, et al. Outcomes of patients with intermediate-risk or poor-risk metastatic renal cell carcinoma who received their first cycle of nivolumab and ipilimumab in the hospital because of symptomatic disease: The MD Anderson Cancer Center experience. Int J Cancer 2021;149:387-93. DOI PubMed

30. McDermott DF, Lee JL, Bjarnason GA, et al. Open-label, single-arm phase II study of pembrolizumab monotherapy as first-line therapy in patients with advanced clear cell renal cell carcinoma. J Clin Oncol 2021;39:1020-8. DOI PubMed PMC

31. Grimm MO, Schmidinger M, Martinez ID, et al. Tailored immunotherapy approach with nivolumab in advanced renal cell carcinoma (TITAN-RCC). Ann Oncol 2019;30:v892. DOI

32. McKay RR, McGregor BA, Xie W, et al. Optimized management of nivolumab and ipilimumab in advanced renal cell carcinoma: a response-based phase II study (OMNIVORE). J Clin Oncol 2020;38:4240-8. DOI PubMed PMC

33. Atkins MB, Jegede $\mathrm{O}$, Haas NB, et al. Phase II study of nivolumab and salvage nivolumab + ipilimumab in treatment-naïve patients (pts) with advanced renal cell carcinoma (RCC) (HCRN GU16-260). J Clin Oncol 2020;38:5006. DOI

34. Lee C, Shah AY, Hsieh JJ, et al. Phase II trial of lenvatinib (LEN) plus pembrolizumab (PEMBRO) for disease progression after PD1/PD-L1 immune checkpoint inhibitor (ICI) in metastatic clear cell renal cell carcinoma (mccRCC). J Clin Oncol 2020;38:5008. DOI

35. Epaillard N, Simonaggio A, Elaidi R, et al. BIONIKK: A phase 2 biomarker driven trial with nivolumab and ipilimumab or VEGFR tyrosine kinase inhibitor (TKI) in naïve metastatic kidney cancer. Bulletin du Cancer 2020;107:eS22-7. DOI PubMed

36. Beuselinck B, Job S, Becht E, et al. Molecular subtypes of clear cell renal cell carcinoma are associated with sunitinib response in the metastatic setting. Clin Cancer Res 2015;21:1329-39. DOI PubMed

37. Choueiri TK, Albiges L, Powles T, Scheffold C, Wang F, Motzer RJ. A phase III study (COSMIC-313) of cabozantinib (C) in combination with nivolumab $(\mathrm{N})$ and ipilimumab (I) in patients (pts) with previously untreated advanced renal cell carcinoma (aRCC) of intermediate or poor risk. J Clin Oncol 2020;38:TPS767. DOI

38. Apolo AB, Nadal R, Girardi DM, et al. Phase I study of cabozantinib and nivolumab alone or with ipilimumab for advanced or metastatic urothelial carcinoma and other genitourinary tumors. J Clin Oncol 2020;38:3672-84. DOI PubMed PMC 
39. Choueiri TK, Bauer TM, Mcdermott DF, et al. Phase 2 study of the oral hypoxia-inducible factor $2 \alpha$ (HIF-2 $\alpha$ ) inhibitor MK-6482 in combination with cabozantinib in patients with advanced clear cell renal cell carcinoma (ccRCC). J Clin Oncol 2021;39:272. DOI

40. Charych DH, Hoch U, Langowski JL, et al. NKTR-214, an engineered cytokine with biased IL2 receptor binding, increased tumor exposure, and marked efficacy in mouse tumor models. Clin Cancer Res 2016;22:680-90. DOI PubMed

41. Diab A, Tannir NM, Bentebibel SE, et al. Bempegaldesleukin (NKTR-214) plus nivolumab in patients with advanced solid tumors: phase I dose-escalation study of safety, efficacy, and immune activation (PIVOT-02). Cancer Discov 2020;10:1158-73. DOI PubMed

42. Tannir NM, Agarwal N, Pal SK, et al. A phase III randomized open label study comparing bempegaldesleukin (NKTR-214) plus nivolumab to sunitinib or cabozantinib (investigator's choice) in patients with previously untreated advanced renal cell carcinoma. $J$ Clin Oncol 2019;37:TPS4595. DOI

43. Dovedi SJ, Elder MJ, Yang C, et al. Design and efficacy of a monovalent bispecific PD-1/CTLA4 antibody that enhances CTLA4 blockade on PD- $1^{+}$activated T cells. Cancer Discov 2021;11:1100-17. DOI PubMed 